\title{
BARRIER AND GUARDRAIL EXTRACTION AND CLASSIFICATION FROM POINT CLOUDS
}

\author{
M. Vidal ${ }^{\mathrm{a}}$, L. Díaz-Vilariño ${ }^{\mathrm{b}}$, P. Arias ${ }^{\mathrm{b}}, \mathrm{J}$. Balado ${ }^{\mathrm{b}}$ \\ ${ }^{a}$ Universidade de Vigo. Escola de Enxeñaría Industrial, Campus universitario de Vigo, As Lagoas, Marcosende, 36310 Vigo, Spain. \\ martvidal@alumnos.uvigo.es \\ ${ }^{\text {b }}$ Universidade de Vigo. Centro de Investigación en Tecnoloxías, Enerxía e Procesos Industriais (CINTECX). Applied \\ Geotechnologies Research Group, Campus universitario de Vigo, As Lagoas, Marcosende, 36310 Vigo, Spain. \{lucia, parias, \\ jbalado\}@uvigo.es
}

\section{Commission II/9}

KEY WORDS: Mobile laser scanning, semantic segmentation, road infrastructure, HD maps, point cloud processing.

\begin{abstract}
:
In the recent years, the modelling of infrastructures has been receiving increasingly attention due to the importance of transport infrastructures for global economy, traffic safety and for the generation of high definition maps, essential to autonomous vehicles. This paper presents a simple method for the segmentation and classification of concrete barriers and guardrails in road surroundings. First steps of the method are aimed to delimit the region of the point cloud outside the driving lanes in which barriers and guardrails are installed. The purpose is to significantly reduce the size of point clouds in order to improve further processing. Then, barrier segmentation and classification are designed as parameter-dependent processes because the geometric features of roads and barriers and guardrails are mostly regulated by norms and standards. Results show a good performance in terms of classification in comparison of other state of the art methods. Better results were obtained for guardrails than for concrete barriers. The method has been tested in a set of point clouds acquired with a Mobile Laser Scanner from conventional roads and highways.
\end{abstract}

\section{INTRODUCTION}

In 2018, more than 25,000 people died in accidents in the European Union. As a result of the high level of road accidents, the European Union has set the goal of zero road deaths by 2050 (DGT, 2019). Road exits are particularly dangerous and costly, as cars can head on clash with other vehicles and infrastructures or fall into areas that are difficult for the emergency services to access. Barriers and guardrails on road edges are the main passive safety element to prevent road exits.

Both the location and the characteristics of guardrails on road edges are established by regulations in each country (Ministerio de Fomento, 2014), and depend on the dangerousness and the road characteristics. Thus, guardrails should be placed at access points to bridges or tunnels, embankments, ravines, rivers, reservoirs, walls, areas with posts, crossings over railroads, roads with daily traffic of more than 10,000 vehicles, and parallel roads with medians of less than $1 \mathrm{~m}$. The guardrail regulations establish the orientation (parallel to road axis and perpendicular to the asphalt), with a minimum distance to obstacles, a minimum length, and a minimum distance from the shoulder. The start/end of the barrier can be done by embedding it in a slope, by folding it or by having an element that absorbs a frontal impact.

Guardrails must be mapped, and monitored periodically, so automating the process is of vital importance. It must be verified that the existing rail guards comply with safety regulations and that their state of conservation is adequate, as they may have been damaged in accidents. In this case, it will be necessary to replace the guardrails. The information acquired can be stored on High Definition (HD) maps (Zang et al., 2018), which contain accurate data of road and surrounding elements, very useful for road management, GPS applications, and even autonomous driving.
Due to the importance of barriers and guardrails for traffic safety and for the generation of HD maps, the objective of this work is to develop an automated method for the extraction and characterization of barriers and guardrails from point clouds acquired from a terrestrial Mobile Laser Scanner (MLS). The paper also deals with the importance of assessing if as-built barriers accomplish with current regulations.

The rest of this paper is organized as follows. Section 2 reviews the state of the art in terms of guardrail and barrier detection in road environments. Section 3 presents an overview of the method. Section 4 analyses the results. And finally, Section 5 is devoted for concluding the work.

\section{RELATED WORK}

There are several approaches to address the problem of detecting guardrails in point clouds, either for the generation of High Definition maps or for autonomous driving. One way to address the problem is through object classification. In (Yang et al., 2017, 2016), elements in point clouds are segmented by shape and individualized. Then, the objects are classified by geometric characteristics. Given guardrail shape and location with respect to the road, many authors consider that a knowledge-based approach is the best option. In (Jiang et al., 2016; Zhu and Guo, 2018), point clouds are structured by scanlines. Corrugated beam guardrail in scanlines are defined as points forming a characteristic curve (in orientation and shape) without continuity with the rest of the environment. In a similar way, roads can be segmented into cross section, where the shape and position of guardrails and barriers are also key elements for their recognition (Weiss and Dietmayer, 2007). Ishida et al. (2013) base their method on conformal geometric algebra to delineate roadsides and classify them into guardrails and curbs. Scharwächter et al. (2014), employ stereoscopic images to locate possible guardrails

Corresponding author 
by means of the Hough transform. These candidates are then geometrically verified. Although most works studying guardrails focus on road environments, these barriers exist in other environments as well. In (Wang, 2019), the characteristics of guardrails on scaffold work platforms are analyzed to determine whether barriers comply with safety regulations.

Recently, Artificial Intelligence (AI) has been tested for guardrail detection. PointNet and PointNet++ can semantically segment guardrails in road environments, both in low density point clouds, typical of autonomous driving (Feng et al., 2019), and in higher density clouds used for road object inventory (Balado et al., 2019). But these approaches based on Neural Networks applied directly to point clouds do not always get the best result for all elements. Guardrails, given their limited number of points within the road environment, are not segmented correctly. Methods with simpler Artificial Neural Networks have shown to obtain better results (Balado et al., 2019). One solution may be to integrate AIbased methods into knowledge-based approaches. In (Matsumoto et al., 2019), points belonging to the ground and points far from the road are eliminated, the remaining points are classified by means of a Convolutional Neural Network.

Regarding to previous approaches, this work presents a method for the detection of barriers and guardrails based on previous knowledge extracted from regulation, used for the definition of parameters involved in the process. In addition, the method does not need the input of the trajectory followed by the vehicle during the acquisition.

\section{METHOD}

Since the objective of this paper is limited to extract and classify barriers and guardrails, the method starts by a sequence of steps (Section 3.1.) aiming to determine the Region of Interest (ROI) (Section 3.2.), defined as the region of the road environment most likely to contain road concrete barriers and guardrails. Points belonging to barriers and guardrails are next segmented and classified according to their geometric features which are previously known and defined by regulations (Section 3.3.).

\subsection{Data pre-processing}

The ultimate purpose of data pre-processing is to define the road centreline, which will be further used to determine the region of interest (ROI).

For this purpose, point clouds are firstly submitted to a filtering based on the number of returns by which just last-return points are further processed. With this process, the size of point cloud is reduced without affecting to points belonging to road infrastructure. Those points eliminated are mostly belonging to vegetation of road surroundings.

Next, points representing road markings are roughly isolated by selecting points with an intensity higher than $80 \%$ and by clustering those points using a density-based spatial clustering (Ester et al, 1996), followed by checking cluster size and scan angle. Figure 1 shows a point cloud submitted to selection by intensity before (a) and after clusterization and selection by continuity and scan angle (b).

The angle formed by road markings with scanner along the trajectory of the vehicle tends to be constant. Therefore, the angle of each cluster is determined, and clusters will be grouped by angle. Longitudinal road markings (markings delimiting driving lanes) will correspond with the most populated groups of clusters.
For conventional roads, three groups of clusters will be obtained: one group placed at a positive angle -road marking at the right side of the vehicle-, another group placed at a negative angle close to zero -centreline- and another group placed at a higher negative angle - road making at the left side of the vehicle-. With this verification, clusters belonging to other elements such as vertical traffic signs are pruned, and main road markings are extracted in a way that they can be used for defining the region of interest for further processing.

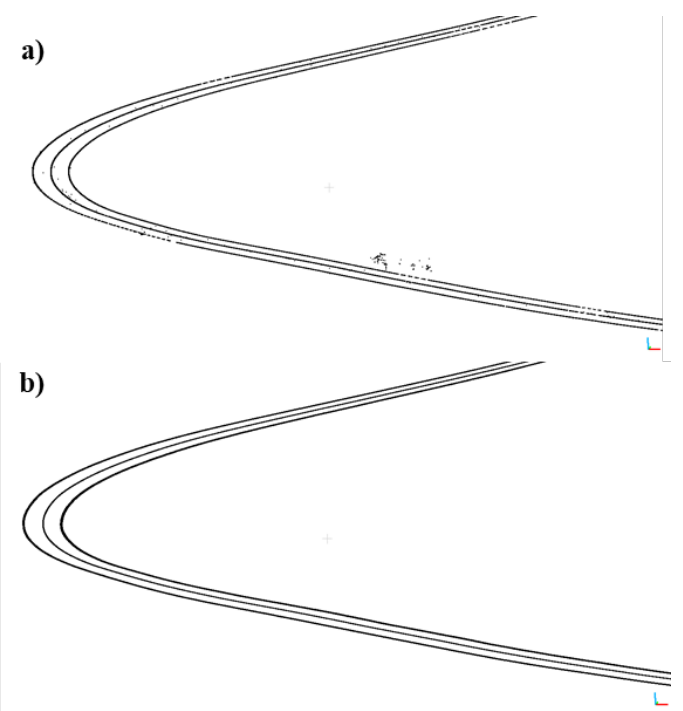

Figure 1. a) points with an intensity higher than $80 \%$, b) points after selection by continuity and scan angle.

\subsection{Definition of the Region of Interest}

In this method, the region of interest is defined as the region of the point cloud outside the driving lanes in which barriers and guardrails are installed (Figure 3).

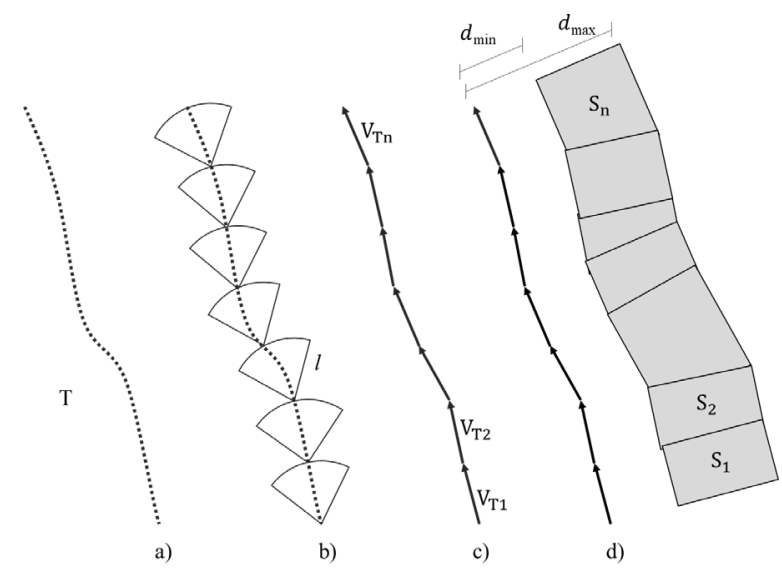

Figure 2. Segmentation from road centreline, top view: a) points belonging to road centreline, b) radius search along trajectory,

c) vectorization, d) point cloud in stretches (adapted from Balado et al, 2017).

For isolating the ROI, points belonging to the road centreline are subsampled by distance $l$, and the centreline is vectorised. The set of points $T$ is transformed into vectors $\mathrm{V}_{\mathrm{T}}=\left(\mathrm{V}_{\mathrm{x}}, \mathrm{V}_{\mathrm{y}}, \mathrm{V}_{\mathrm{z}}\right)$, with $\left\|\mathrm{V}_{\mathrm{T}}\right\| \approx l$ and let $\mathrm{V}_{\mathrm{Ti}}=\mathrm{T}_{\mathrm{i}}(\mathrm{i} l+1)-\mathrm{T}_{\mathrm{i}}(\mathrm{i} l)$ (Figure 2). The point cloud $\mathrm{P}=\left(\mathrm{X}_{\mathrm{P}}, \mathrm{Y}_{\mathrm{P}}, \mathrm{Z}_{\mathrm{P}}\right)$ is segmented in stretches $\mathrm{S}=$ $\left\{S_{1}, S_{2} \ldots S_{n}\right\}$ perpendicular of each vector at the final point $S_{i}=$ 
$\left\{\mathrm{P}:<\perp \mathrm{T}_{\mathrm{i}}(\mathrm{i} l+1)\right\}$. In this way, the ROI is the zone belonging to each stretch $\mathrm{C}_{\mathrm{ROIi}} \subset \mathrm{S}_{\mathrm{i}}$ (Figure 2.d) taking into account a minimum and maximum distance $d$ from the road centreline. Figure 3 shows the points belonging to the region of interest in grey.

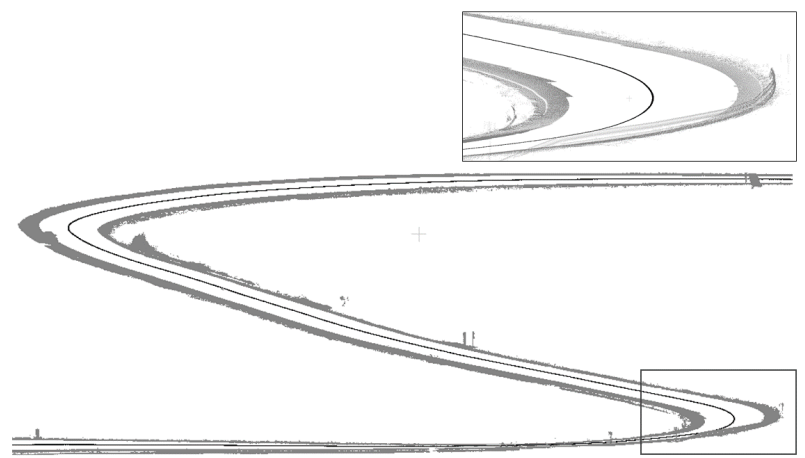

Figure 3. Points belonging to the ROI (in grey) and to the road centreline (in black).

\subsection{Barrier and guardrail extraction and classification}

The barrier and guardrail extraction starts by classifying the points of the ROI in ground and off-ground points. This can be made by calculating the plane defining each stretch $S_{i}$ and containing the corresponding vector centreline $V_{T i}$. Off-ground points will be those points with vertical distance to the plane, higher than a certain threshold (Figure 4, in blue).

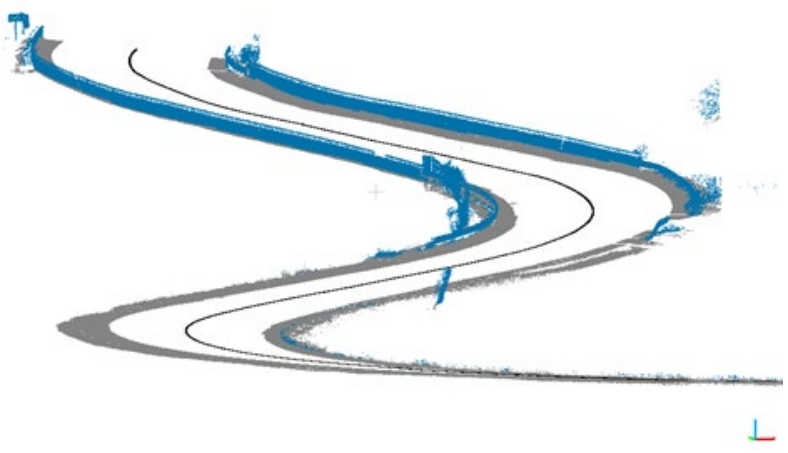

Figure 4. Ground points in grey, off-ground points in blue.

Next, off-ground points are clustered with DBSCAN, and clusters are further studied in order to extract barriers and/or guardrails. If the cluster has a longitudinal length higher than $l$, clusters are subdivided in stretches of $1 \mathrm{~m}$ in order to study them individually. Then, clusters are classified as barriers and guardrails according to their point density, and their maximum and minimum height. Figure 5 shows how points belonging to barriers and guardrails are segmented (visualized in blue).

Final step aims to classify points belonging to barriers and guardrails in two classes according to their geometry. Both elements are often installed together. Since barriers and guardrails dimensions are defined by conventions and norms, these parameters are previously known. In this method, barriers and guardrails are again longitudinally segmented, and stretches classified into barriers or guardrails based on number of points, maximum and minimum height and connectivity with ground. Figure 6 shows two examples in which concrete barrier (in violet) and guardrail (in green) classification is performed correctly.

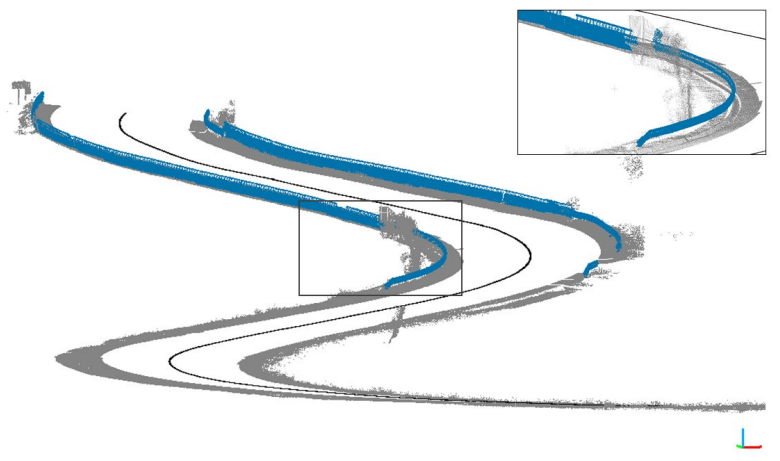

Figure 5. Points classified as barriers or guardrails (in blue), and discarded points (in grey).

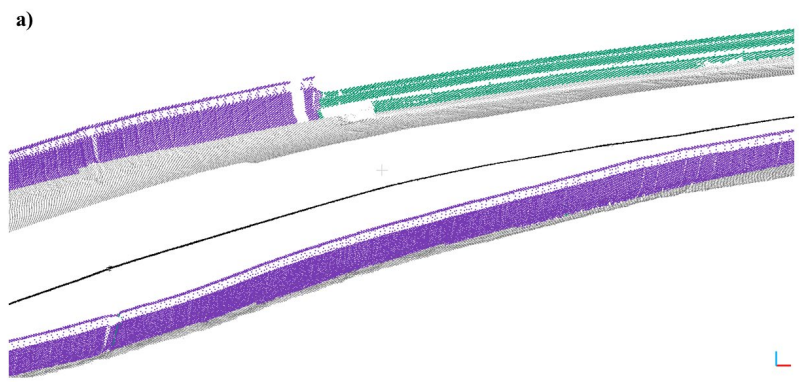

b)

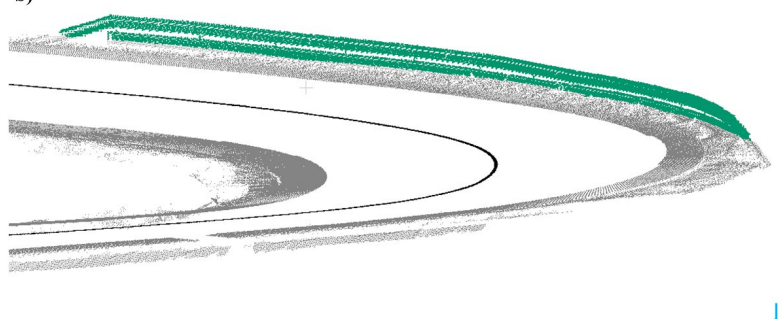

Figure 6. Concrete barriers highlighted in violet, and double guardrails visualized in green.

\section{RESULTS}

\subsection{Data}

In addition to the point cloud used to describe the method, a set of six case studies were also used to test the performance (Figure 7). Point clouds have been acquired with the MLS LYNX Mobile Mapper of Optech (Puente et al, 2013) and they are georeferenced. Point clouds contain a total of 82.7 million points and a $9.4 \mathrm{~km}$ of longitudinal section (Table 1). All processes have been programmed in Python and the code was run on an Intel Core i5 CPU $1.8 \mathrm{GHz}$ with $6 \mathrm{~GB}$ RAM. 
a)

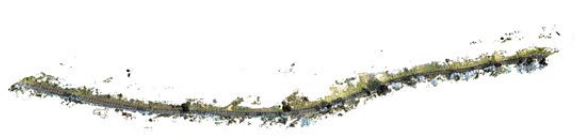

d)

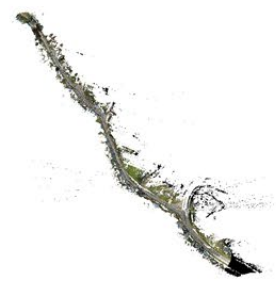

b)

)

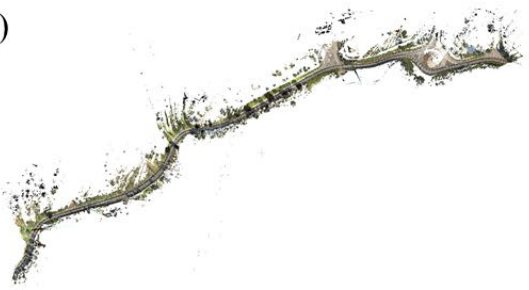

e)

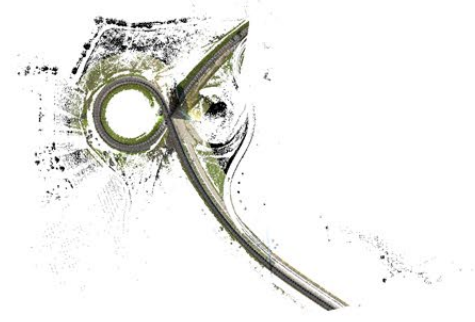

c)

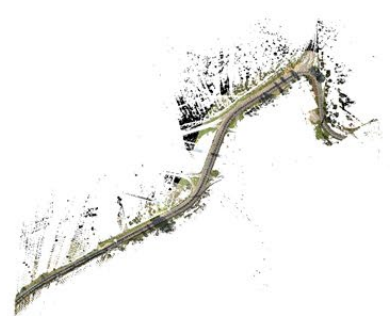

f)

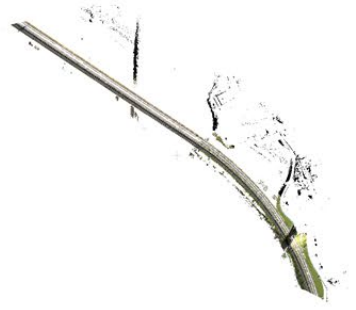

Figure 7. Top view of point clouds acquired in: a)-d) conventional roads, e)-f) highways.

\begin{tabular}{cccc}
\hline & Typology & Points (M) & $\begin{array}{c}\text { Longitude } \\
(\mathrm{km})\end{array}$ \\
\hline $\mathbf{0}$ & Conventional & 12.5 & 2.2 \\
\hline $\mathbf{a}$ & Conventional & 14.6 & 1.3 \\
\hline $\mathbf{b}$ & Conventional & 15.6 & 1.5 \\
\hline $\mathbf{c}$ & Conventional & 12.5 & 1.2 \\
\hline $\mathbf{d}$ & Conventional & 13.2 & 1.1 \\
\hline $\mathbf{e}$ & Highway & 7.8 & 1.1 \\
\hline $\mathbf{f}$ & Highway & 6.5 & 1.0 \\
\hline TOTAL & & $\mathbf{8 2 . 7}$ & $\mathbf{9 . 4}$ \\
\hline
\end{tabular}

Table 1. Characteristics of point clouds used as case study.

\subsection{Results}

As previously mentioned, data pre-processing starts by selecting last-return points and filtering those points by intensity using a threshold of $80 \%$. This value was empirically determined since it was demonstrated to be enough for segmenting most of points belonging to road markings. After that, those points were clustered and those clusters with a size lower than 3 were discarded. Then, the angle for each cluster was calculated and clusters defining the centreline were automatically determined.

The ROI was isolated by analysing stretches of $l=5 \mathrm{~m}$, and $\mathrm{d}_{\min }$ $=2 \mathrm{~m}, \mathrm{~d}_{\max }=7 \mathrm{~m}$ (for conventional roads) and $\mathrm{d}_{\max }=9 \mathrm{~m}$ (for highways), and a maximum height $=2 \mathrm{~m}$. With this process, the total number of points was reduced from 82.7 million of points to 3.4 million of points.

Barrier and guardrail segmentation was performed by subdividing the ROI into $1 \mathrm{~m}$ long stretches. Point clouds were clustered, and clusters wer classified of interest if they were composed of a minimum of 200 points, and if their minimum and maximum height were $0.15 \mathrm{~m}$ and $1 \mathrm{~m}$, respectively. Points of interest were finally classified into barriers and guardrails depending on the neighbouring size, maximum height and floor connection. Concrete barriers have a higher neighbouring size, a higher maximum height and connection with floor. Results are summarized in Table 2 and Table 3.

\begin{tabular}{cccccccccc}
\hline \multirow{2}{*}{ Point cloud } & \multicolumn{3}{c}{ Concrete Barriers } & \multicolumn{3}{c}{ Guardrails } & \multicolumn{3}{c}{$\begin{array}{c}\text { Concrete Barriers \& } \\
\text { Guardrails }\end{array}$} \\
\cline { 2 - 10 } & Precision & Recall & F1 & Precision & Recall & F1 & Precision & Recall & F1 \\
\hline $\mathbf{0}$ & 0.99 & 0.77 & 0.86 & 0.78 & 0.88 & 0.83 & 0.91 & 0.80 & 0.85 \\
\hline $\mathbf{a}$ & 0.89 & 0.61 & 0.73 & 0.89 & 0.95 & 0.92 & 0.89 & 0.87 & 0.88 \\
\hline $\mathbf{b}$ & - & - & - & 0.97 & 0.96 & 0.97 & 0.97 & 0.96 & 0.97 \\
\hline $\mathbf{c}$ & - & - & - & 1.00 & 0.67 & 0.80 & 1.00 & 0.67 & 0.80 \\
\hline $\mathbf{d}$ & 0.59 & 0.72 & 0.65 & 1.00 & 0.85 & 0.92 & 0.93 & 0.84 & 0.88 \\
\hline $\mathbf{e}$ & 0.72 & 0.38 & 0.50 & 0.84 & 0.81 & 0.82 & 0.81 & 0.65 & 0.72 \\
\hline $\mathbf{f}$ & 0.69 & 0.77 & 0.72 & 0.99 & 0.56 & 0.71 & 0.78 & 0.67 & 0.72 \\
\hline TOTAL & $\mathbf{0 . 7 6}$ & $\mathbf{0 . 6 7}$ & $\mathbf{0 . 7 1}$ & $\mathbf{0 . 9 1}$ & $\mathbf{0 . 7 5}$ & $\mathbf{0 . 8 3}$ & $\mathbf{0 . 8 5}$ & $\mathbf{0 . 7 2}$ & $\mathbf{0 . 7 8}$ \\
\hline
\end{tabular}

Table 2 shows the classification results through a Precision and Recall analysis. 


\begin{tabular}{cccccc}
\hline Point cloud & Pre-processing (s) & ROI extraction (s) & $\begin{array}{c}\text { Barriers } \\
\text { segmentation (s) }\end{array}$ & $\begin{array}{c}\text { Barriers } \\
\text { classification (s) }\end{array}$ & Total time (min) \\
\hline $\mathbf{0}$ & 110.4 & 443.7 & 64.5 & 20.3 & 10.6 \\
\hline $\mathbf{a}$ & 128.7 & 663.3 & 74.1 & 16.7 & 14.7 \\
\hline $\mathbf{b}$ & 171.3 & 1041.5 & 255.3 & 14.9 & 24.7 \\
\hline $\mathbf{c}$ & 120.5 & 656.4 & 178.6 & 12.0 & 16.1 \\
\hline $\mathbf{d}$ & 133.4 & 641.7 & 136.1 & 14.9 & 15.4 \\
\hline $\mathbf{e}$ & 32.1 & 366.8 & 125.6 & 22.7 & 9.1 \\
\hline $\mathbf{f}$ & 26.7 & 285.9 & 185.2 & 29.9 & 8.8 \\
\hline
\end{tabular}

Table 3 shows the processing time for each step of the method.

For the evaluation of the method, a quantitative analysis based on precision and recall indices was carried out. The comparison was performed using the point clouds manually segmented and classified as ground truth.

Results show a better performance for guardrails than for concrete barriers, both in terms of precision and recall. This means that for guardrails a low proportion of False Positives and False Negatives was obtained. The low recall in case study e) for concrete barriers was due to the non-classified barriers belonging to the opposite-sense lanes of the highway. It should be noticed that the point clouds used as case studies were point clouds acquired in a one-sense driving. This means that, especially for highways, there was a higher number of false negatives belonging to those barriers on the opposite-sense lanes that were not complete acquired, and consequently, that were not correctly classified. In this case, results are expected to improve if case studies are captured from the two-sense driving lanes. Figure 8 shows an example of a wrong classified area in case study e). On the right side (Figure 8.b), there is a concrete barrier wrongly classified as guardrail (in green), because it corresponds to the other sense driving of the highway and it is partially occluded in the point cloud.
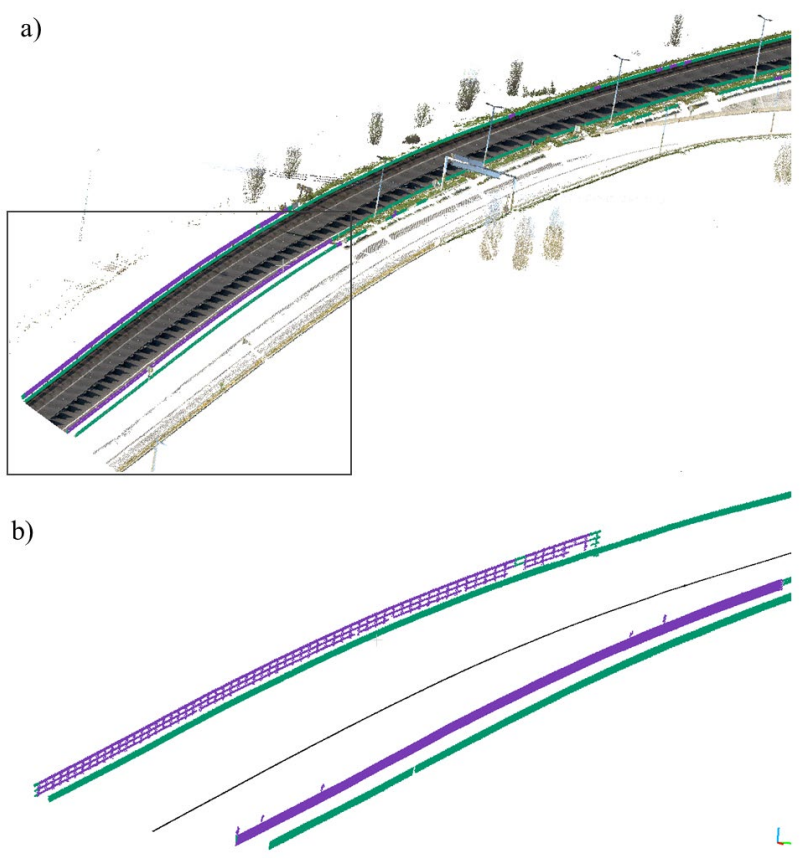

Figure 8. A partial view of results obtained for case study e (concrete barriers in violet, and guardrails in green).
Although this method is parameter-dependent, results are slightly better than those obtained by other methods based on Deep Learning techniques such as Point Net (Balado et al, 2019). Since road and, barrier and guardrail dimensions are mostly standardized and regulated by norms, parameters can be predefined according to the type of road. This previous knowledge would also enable to check if as-built barriers and guardrails accomplish with current regulations.

With regard to the processing time (Table 3), it should be noticed that case studies were processed with an Intel Core i5 CPU 1.8 GHz with 6 GB RAM.

\section{CONCLUSIONS}

This work specifically addresses the segmentation and classification of concrete barriers and guardrails installed in different typologies of roads. For this purpose, a simple datadriven approach is presented. The method starts by a set of steps aimed to delimit the region of the point cloud outside the driving lanes in which barriers and guardrails are installed. This reduces the size of point cloud in a $96 \%$, making the segmentation and classification of barriers and guardrails more effective. Then, points are segmented and classified following a sequence of steps based on geometrical and dimensional parameters. This can be done in this way because the location and the dimensions of these road protection elements are mostly regulated and standardized. Although the method is simple and parameter-dependent, results show a good performance in terms of classification, and in comparison of other state of the art methods.

Future work will extend the method to other road typologies and barriers, and results will be used to analyse if these protection elements are correctly installed and maintained. Combination of knowledge-based methods with AI will be also explored.

\section{ACKNOWLEDGEMENTS}

Authors would like to thank to the Xunta de Galicia given through human resources grant (ED481B-2019-061, ED481D 2019/020), the Ministerio de Ciencia, Innovación y Universidades -Gobierno de España- (RTI2018-095893-B-C21). This project has received funding from the European Union's Horizon 2020 research and innovation programme under grant agreement No 769255. This document reflects only the views of the author(s). Neither the Innovation and Networks Executive Agency (INEA) or the European Commission is in any way responsible for any use that may be made of the information it contains. The statements made herein are solely the responsibility of the authors. 


\section{REFERENCES}

Balado, J., Díaz-Vilariño, L., Arias, P., Soilán, M. 2017. Automatic building accessibility diagnosis from point clouds. Automation in Construction, 82, 00. 103-111, 10.1016/j.autcon.2017.06.026

Balado, J., Martínez-Sánchez, J., Arias, P., Novo, A., 2019. Road Environment Semantic Segmentation with Deep Learning from MLS Point Cloud Data. Sensors. https://doi.org/10.3390/s19163466

DGT, 2019. En 2018 murieron 25.100 europeos en accidentes [WWW Document]. DGT Magazine. URL http://revista.dgt.es/es/noticias/internacional/2019/0411unioneuropea-informe-mortalidad-2018.shtml\#.XnUBuohKi70 (accessed 3.23.20).

Ester, M., Kriegel, H.P., Sander, J., Xu, X., 1996. A densitybased algorithm for discovering clusters in large spatial databases with noise. Proceedings of the Second International Conference on Knowledge Discovery and Data Mining (KDD-96). AAAI Press. pp. 226-231

Ministerio de Fomento - Gobierno de España, 2014. Orden Circular 35/2014 Sobre Criterios De Aplicación De Sistemas De Contención De Vehículos.

Feng, Z., Zhang, S., Kunert, M., Wiesbeck, W., 2019. Point Cloud Segmentation with a High-Resolution Automotive Radar, in: AmE 2019 - Automotive Meets Electronics; 10th GMMSymposium. pp. 1-5.

Ishida, H., Meguro, J., Kojima, Y., Naito, T., 2013. 3D Road Boundary Detection Using Conformal Geometric Algebra. IPSJ Transactions on Computer Vision and Applications 5, 176-182. https://doi.org/10.2197/ipsjtcva.5.176

Jiang, Y., He, B., Liu, L., Ai, R., Lang, X., 2016. Effective and robust corrugated beam guardrail detection based on mobile laser scanning data, in: 2016 IEEE 19th International Conference on Intelligent Transportation Systems (ITSC). pp. 1540-1545. https://doi.org/10.1109/ITSC.2016.7795762

Matsumoto, H., Mori, Y., Masuda, H., 2019. Extraction and shape reconstruction of guardrails using mobile mapping data. ISPRS - International Archives of the Photogrammetry, Remote Sensing and Spatial Information Sciences XLII-2/W13, 10611068. https://doi.org/10.5194/isprs-archives-XLII-2-W13-10612019

Puente, I.; González-Jorge, H.; Martínez-Sánchez, J.; Arias, P. Review of mobile mapping and surveying technologies. Meas. J. Int. Meas. Confed. 2013, 46, 2127-2145.

Scharwächter, T., Schuler, M., Franke, U., 2014. Visual guard rail detection for advanced highway assistance systems, in: 2014 IEEE Intelligent Vehicles Symposium Proceedings. pp. 900-905. https://doi.org/10.1109/IVS.2014.6856573

Wang, Q., 2019. Automatic checks from 3D point cloud data for safety regulation compliance for scaffold work platforms. Automation in Construction 104, 38-51. https://doi.org/https://doi.org/10.1016/j.autcon.2019.04.008

Weiss, T., Dietmayer, K., 2007. Automatic Detection of Traffic Infrastructure Objects for the Rapid Generation of Detailed Digital Maps using Laser Scanners, in: 2007 IEEE Intelligent Vehicles Symposium. pp. 1271-1277. https://doi.org/10.1109/IVS.2007.4290293
Yang, B., Dong, Z., Liu, Y., Liang, F., Wang, Y., 2017. Computing multiple aggregation levels and contextual features for road facilities recognition using mobile laser scanning data. ISPRS Journal of Photogrammetry and Remote Sensing 126, 180-194. https://doi.org/10.1016/j.isprsjprs.2017.02.014

Yang, B., Liu, Y., Liang, F., Dong, Z., 2016. Using mobile laser scanning data for features extraction of high accuracy driving maps, ISPRS - International Archives of the Photogrammetry, Remote Sensing and Spatial Information Sciences. https://doi.org/10.5194/isprs-archives-XLI-B3-433-2016

Zang, A., Chen, X., Trajcevski, G., 2018. High Definition Maps in Urban Context. SIGSPATIAL Special 10, 15-20. https://doi.org/10.1145/3231541.3231546

Zhu, H., Guo, B., 2018. A Beam Guardrail Detection Algorithm Using Lidar for Intelligent Vehicle. https://doi.org/10.1109/CYBER.2018.8688363 